\title{
Tuning Electronic Structures of BN and C Double-Wall Hetero-Nanotubes
}

\author{
Xueran Liu, Meijun Han, Xinjiang Zhang, Haijun Hou, Shaoping Pang, and Qisheng Wu \\ School of Materials Engineering, Yancheng Institute of Technology, Yancheng, Jiangsu 224051, China \\ Correspondence should be addressed to Shaoping Pang; psp800@126.com and Qisheng Wu; qishengwu@ycit.cn
}

Received 8 April 2015; Revised 15 June 2015; Accepted 2 July 2015

Academic Editor: Dan Xia

Copyright (C) 2015 Xueran Liu et al. This is an open access article distributed under the Creative Commons Attribution License, which permits unrestricted use, distribution, and reproduction in any medium, provided the original work is properly cited.

First principle calculations based on density functional theory with the generalized gradient approximation were carried out to investigate the energetic and electronic properties of carbon and boron nitride double-wall hetero-nanotubes (C/BN-DWHNTs) with different chirality and size, including an armchair $(n, n)$ carbon nanotube $(\mathrm{CNT})$ enclosed in $(m, m)$ boron nitride nanotube $(\mathrm{BNNT})$ and a zigzag $(n, 0)$ CNT enclosed in $(m, 0)$ BNNT. The electronic structure of these DWHNTs under a transverse electric field was also investigated. The ability to tune the band gap with changing the intertube distance $\left(d_{i}\right)$ and imposing an external electric field $(F)$ of zigzag DWHNTs provides the possibility for future electronic and electrooptic nanodevice applications.

\section{Introduction}

Carbon nanotubes (CNTs) play a very important role in nanodevice applications due to their novel properties. by comparison with CNTs, boron nitride nanotubes (BNNTs) are formed with similar structures, however, of very distinctive properties [1]. Of similar crystalline structure, hexagonal boron nitride (h-BN) has been considered as a potential substrate material for graphene $[1,2]$. Recently, the structures of bilayer and trilayer graphene/h-BN have been reported with tunable band gaps for electronic device applications [35].

Hexagonal boron nitride shares similar crystalline structure with graphene, and it is slightly lattice-mismatched from graphene by about $1.5 \%$, which implies that it is possible to form hetero-nanostructures. BNNTs' growth on CNT has successfully been applied to nanowires [6]. Several studies have also been conducted on BN-coated CNTs $[7,8]$. The BNNT around the CNTs effectively helps to protect them and makes them more stable; for example, oxidation degradation of CNTs is reduced by coating with BNNTs, and the thermal stability of CNT@BNNTs is far superior to CNTs [9].

An efficient way to modify the band gap of nanotubes is to apply an external electric field $F[10]$. The response of the nanotube to $F$ is of interest for studying its future application, such as that in logic gates, static memory cells, and sensor devices [11-13]. Ab initio calculation showed that band gaps of both CNTs and BNNTs can be greatly reduced by a transverse electric filed [14, 15]. An intriguing question to answer is whether external electric fields can also be an efficient way to modulate the electronic properties of C/BN-DWHNTs.

However, a first-principles study on the stability as well as the electronic properties modulated with $d_{i}$ and $F$ of C/BN-DWHNTs is not available. To fill the deficiency, in this work, we performed a series of first-principles calculations to study the coaxial CNT@BNNT and to examine the electric field shielding effect of BNNT on the inside CNT. We calculated the band structures of coaxial CNT@BNNT consisting of armchair and zigzag CNT cores and BNNT sheaths, focusing on the band structure variations with $d_{i}$ and $F$. The relative insensitivity of armchair CNT@BNNT to $F$, at least for the few cases considered here, suggests that zigzag CNT@BNNT would be a suitable candidate for double-wall hetero-nanotubes devices.

\section{Calculation Methods}

The geometric structure optimization and calculation of the related electrical properties of the CNT@BNNT with no $F$ were conducted using SIESTA [16, 17] and adopted normconserving nonlocal pseudopotentials for the atomic core. 


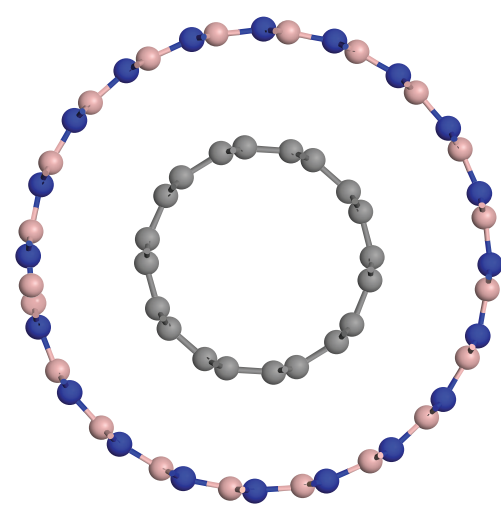

(A)

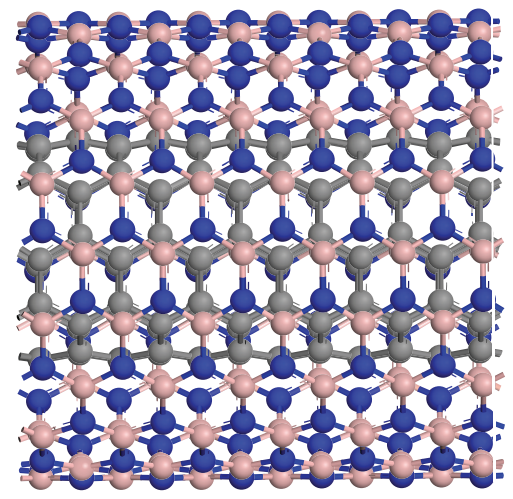

(B)

(a)

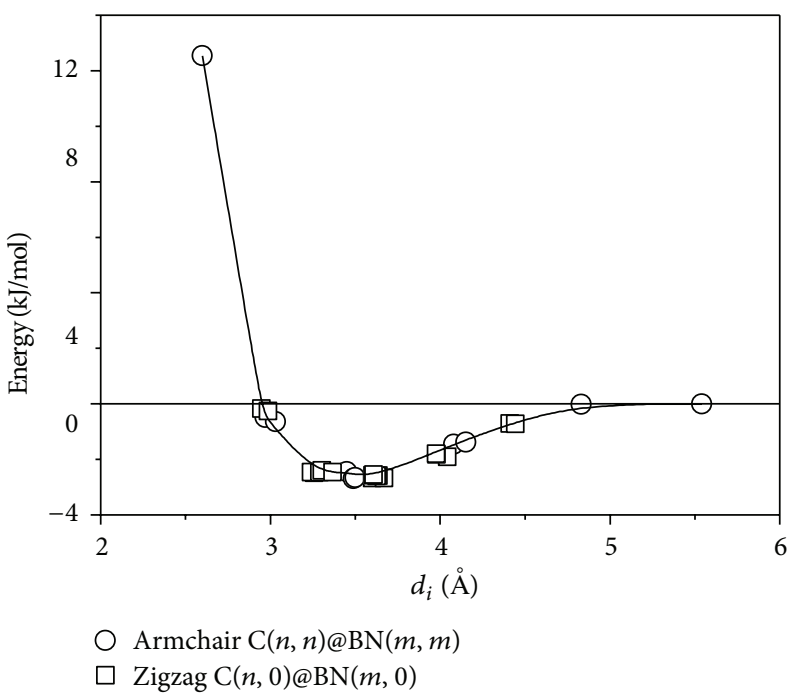

(b)

FIGURE 1: Structure of the optimized CNT $(5,5) @ B N N T(10,10)$ : (a) top view (A) and side view (B). (b) The formation energy $E_{f}$ variation versus intertube distance $d_{i}$. $\bigcirc$ and $\square$ are the formation energy of calculated armchair and zigzag DWHNTs, respectively. The solid line was plotted in eyes.

The Perdew, Burke, and Ernzerhof (PBE) form generalized gradient approximation (GGA) corrections were employed for the exchange-correlation potential energy [18]. The atomic orbital basis set employed throughout was double- $\zeta$ plus polarization functions (DZP). Periodic boundary condition along the axis was employed for nanotubes. Brillouin zones were sampled by a set of $k$-points grid $(1 \times 1 \times 8)$ according to the Monkhorst-Pack approximation [19].

When electric field was imposed, the calculation was using density functional theory available in DMol3 code [20, 21]. The PBE function [22] of GGA was used to calculate the exchange-correlation potential energy, the all electron approach was used, and the orbit population parameter smearing was set at 0.0005 a.u.

Our models were constructed within a tetragonal super cell with lattice constants of $a$ and $b$ equaling $40 \AA$ to avoid the interaction between two adjacent nanotubes and $c$, the lattice constant in $z$ direction along the tube axis, equaling one-dimensional (1D) lattice parameter of the nanotubes.
The tube was taken along the $z$ direction and the circular cross section was lying in the $(x, y)$ plane. Both the CNT and BNNTs structures were fully optimized until the force on each atom was less than $0.005 \mathrm{eV}^{-1}$ during relaxation.

The van der Waals interactions are very important in twodimensional materials [23], especially in layered structures. The van der Waals force has obvious effect on the adsorption energy and adsorption position and height [24-26]. In this paper, the distances of the CNT and BNNT are fixed, and the optimization does not change the C/BN-DWHNTs structures. The van der Waals interactions could increase the value of $E_{f}$, but they should not affect the electronic properties of C/BN-DWHNTs [10].

\section{Results and Discussion}

Two types of C/BN-DWHNTs were investigated, zigzag and armchair. The zigzag C/BN-DWHNTs are studies that include $\operatorname{CNT}(n, 0) @ \operatorname{BNNT}(m, 0)(n=6-10, m=14-20)$. 


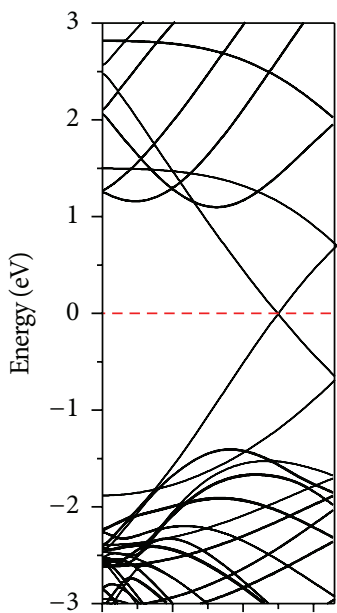

(a)

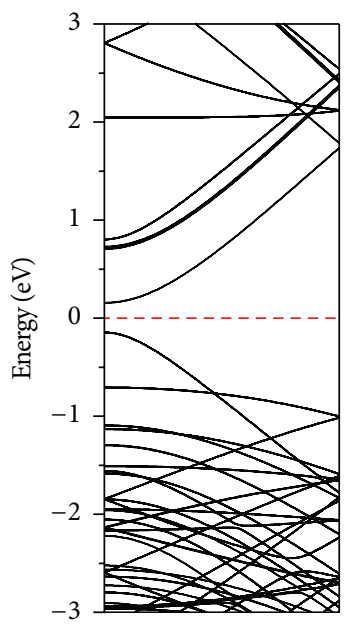

(e)

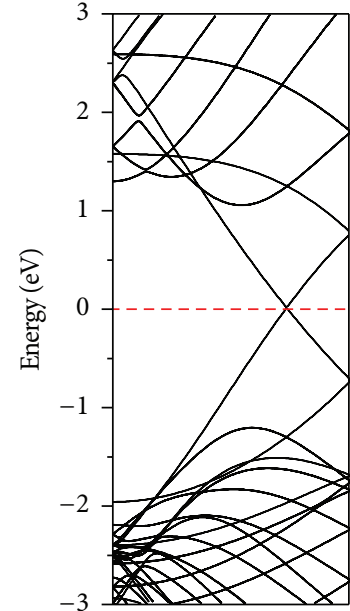

(b)

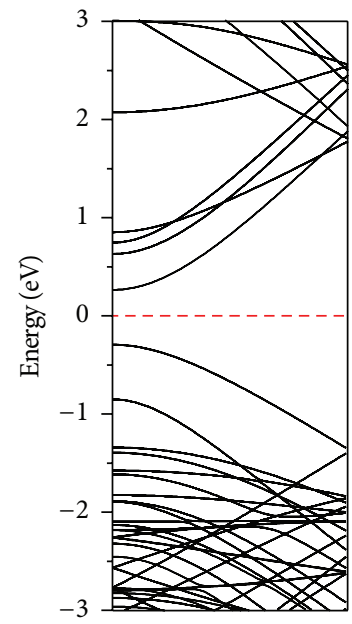

(f)

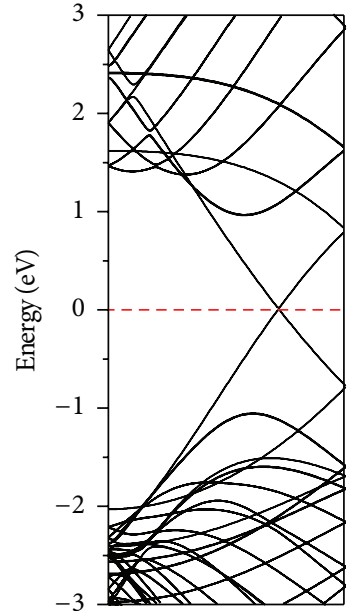

(c)

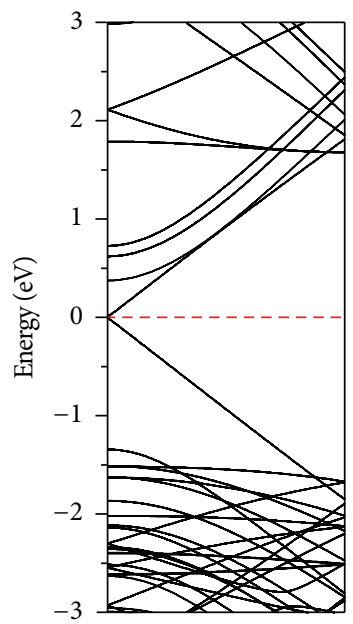

(g)

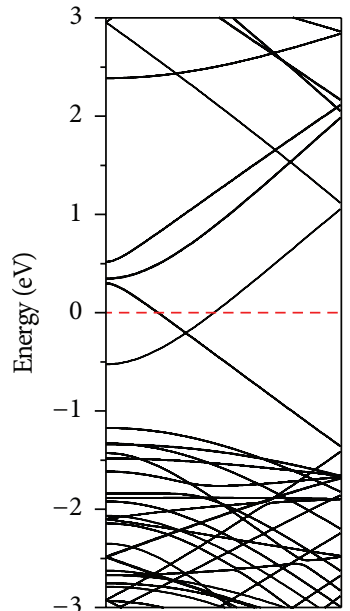

(d)

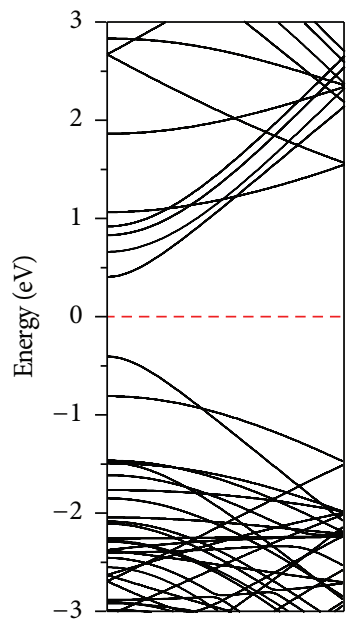

(h)

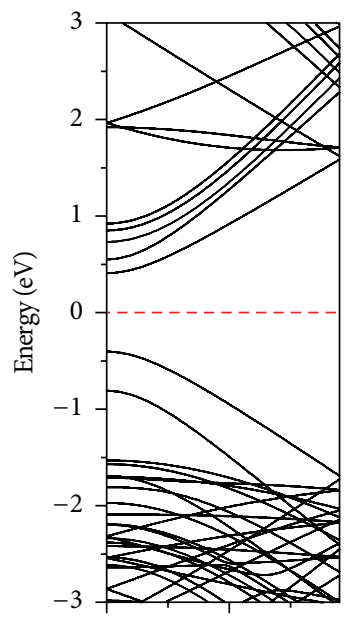

(i)

Figure 2: Band structures of (a) armchair CNT(5, 5)@BNNT(10, 10), (b) armchair CNT(6, 6)@BNNT(11, 11), (c) armchair CNT(7, 7)@BNNT(12, 12), (d) zigzag CNT(6,0)@BNNT(15, 0), (e) zigzag CNT(7, 0)@BNNT(16, 0), (f) zigzag CNT(8, 0)@BNNT(17, 0), (g) zigzag CNT(9, 0)@BNNT(18, 0), (h) zigzag CNT(10, 0)@BNNT(19, 0), and (i) zigzag CNT(11, 0)@BNNT(20, 0). 


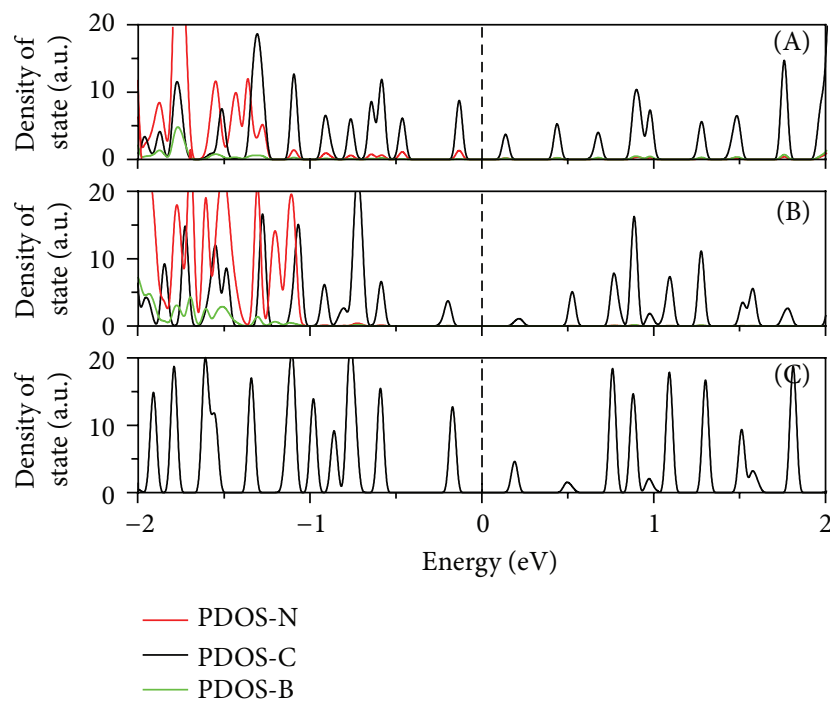

(a)

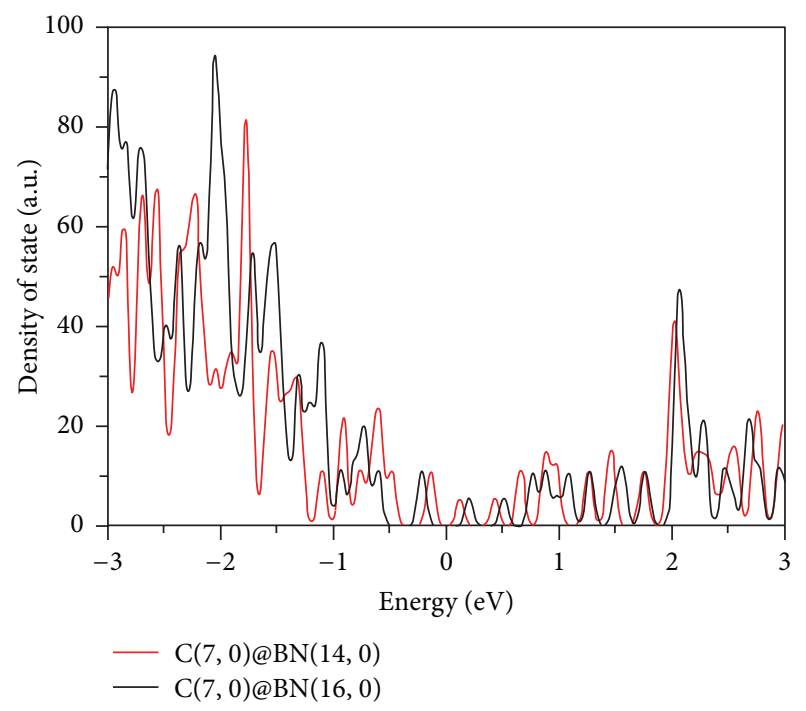

(b)

Figure 3: (a) (A) Partial density of state of zigzag CNT(7, 0)@BNNT(14, 0), (B) partial density of state of zigzag CNT(7, 0)@BNNT(16, 0), and $(\mathrm{C})$ density of state of single-wall CNT $(7,0)$. The Fermi level lies at $0 \mathrm{eV}$ (dash line). (b) Density of state of CNT( 7,0$) @ B N N T(14,0)$ and CNT(7, 0)@BNNT(16, 0) DWHNTs.

The armchair C/BN-DWHNTs considered are $\mathrm{CNT}(n$, $n) @ \operatorname{BNNT}(m, m)(n=5-7, m=8-13)$. Figure 1(a) gives an illustration of the coaxial armchair $\operatorname{CNT}(5,5)$ inside armchair $\operatorname{BNNT}(10,10)$, the left (right) panel for the top (lateral) view of the initial structure.

The calculated covalent bond lengths of the various $\mathrm{C} / \mathrm{BN}-\mathrm{DWHNT}$ in the fully optimized structures are listed in Table 1. For any C/BN-DWHNTs, we define the binding energy per atom as $E_{b}=\left[E_{t}^{@}-x E^{\mathrm{C}}-y\left(E^{\mathrm{B}}+E^{\mathrm{N}}\right)\right] /(x+y)$, where $E^{\mathrm{C}}, E^{\mathrm{B}}$, and $E^{\mathrm{N}}$ are the energy of isolated carbon, boron, and nitride atoms, respectively. $E_{t}^{@}$ is the total energy of a C/BN-DWHNT, $x$ is the number of $\mathrm{C}$ atoms, and $y$ is the number of $\mathrm{B}$ and $\mathrm{N}$ atoms. The formation energy $E_{f}\left(E_{f}=\right.$ $\left.E_{b}^{@}-E_{b}^{\mathrm{C}}-E_{b}^{\mathrm{BN}}\right)$ of each C/BN-DWHNT is also calculated, in which the $E_{b}^{\mathrm{C}}$ and $E_{b}^{\mathrm{BN}}$ are the binding energy of free standing CNT and BNNT, respectively.

The stability of C/BN-DWHNTs is determined by the interaction force between the inner and outer nanotubes, as shown in Table 1 . The formation energy $E_{f}$ varying with $d_{i}$ was plotted in Figure 1(b). When $d_{i}=\sim 2.60 \AA, E_{f}$ is positive, which means the free standing CNT and BNNT are favorite in energy. When $d_{i}=\sim 3.5 \AA$, the armchair C/BNDWHNTs have the lowest binding energy and formation energy, meaning that the armchair C/BN-DWHNTs at $d_{i}=$ $\sim 3.5 \AA$ are more possible to exist. This result is in agreement with the literature [27], in which Yuan and Liew have studied the coaxial CNT@BNNT nanocables and found that the optimal intertube distances between inner $\mathrm{C}$ tube and the outer BN are about $3.5 \AA$ for armchair nanocables.

The distance $d_{i}$ of the armchair C/BN-DWHNTs can be approximately evaluated by the expression as $d_{i}=3\left(m a_{\mathrm{B}-\mathrm{N}}-\right.$ $\left.n a_{\mathrm{C}-\mathrm{C}}\right) / 2 \pi$; here $a_{\mathrm{B}-\mathrm{N}}$ and $a_{\mathrm{C}-\mathrm{C}}$ are the bond lengths of outer BNNT and inner CNT, respectively. Let $d_{i}=3.5 \AA$; the possible stable structures are $\mathrm{CNT}(n, n) @ \mathrm{BNNT}(m, m)$ $(m-n=5)$, for example, CNT $(5,5) @ B N N T(10,10), \mathrm{CNT}(6$, 6)@BNNT(11, 11), and $\operatorname{CNT}(7,7) @ B N N T(12,12) \operatorname{DWHNTs}$ (see Table 1(a)). Taking the armchair CNT $(5,5) @ B N N T(m$, $m)(m=8-13)$ nanotubes as examples, the covalent bond lengths in the fully optimized CNT and BNNT are 1.43 and $1.45 \AA$, respectively. When $d_{i}=\sim 2.60 \AA$, the bond length of inner CNT was suppressed to $1.385 \AA$ and the bond length of outer BNNT was extended to $1.540 \AA$ in the perpendicular direction. This result indicates the occurrence of greater repulsive interactions between the inner CNT and the outer BNNT.

The calculated covalent bond lengths of the various zigzag DWHNTs in fully optimized structures are listed in Table 1 (b). When $d_{i}=\sim 3.6 \AA$, the zigzag C/BN-DWHNTs have the lowest binding energy and formation energy. It means that the C/BN-DWHNTs at $d_{i}=\sim 3.6 \AA$ are more possible to exist. For zigzag configurations, the distance $d_{i}$ is as follows: $d=\sqrt{3}\left(m a_{\mathrm{B}-\mathrm{N}}-n a_{\mathrm{C}-\mathrm{C}}\right) / 2 \pi$; letting $d=3.6 \AA$, the possible stable structures are $\mathrm{CNT}(n, 0) @ \mathrm{BNNT}(m, 0)$ $(m-n=9)$, for example, $\operatorname{CNT}(6,0) @ B N N T(15,0), \operatorname{CNT}(7$, $0) @ B N N T(16,0)$ DWHNTs.

The calculated band structures of C/BN-DWHNTs are shown in Figure 2. The armchair C/BN-DWHNTs are metallic, with the lowest conduction band and the highest valence band crossing over the Fermi level at $\sim 2 / 3$ along $\Gamma-Z$ direction in reciprocal space. The electric band structure near the Femi level is very similar to the CNTs because the lowest conduction band and the highest valence band are determined by the carbon atoms. We also found that changing $d_{i}$ cannot be an efficient way to open the band gap for armchair C/BNDWHNTs.

The calculated band gaps $\left(E_{g}\right)$ of the single-wall CNT $(n$, 0 ) are $0.19,0.58,0.04,0.78$, and $0.87 \mathrm{eV}$ for $n=7-11$, 


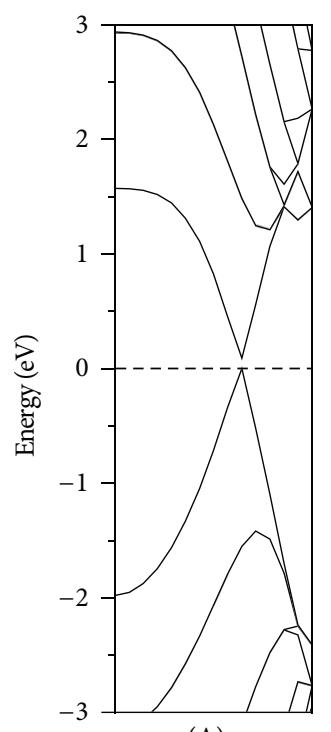

(A)

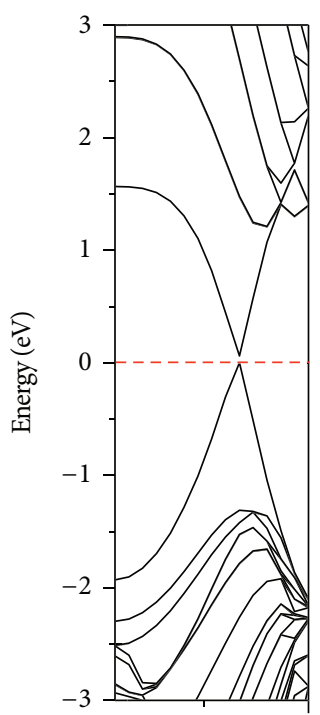

(A)

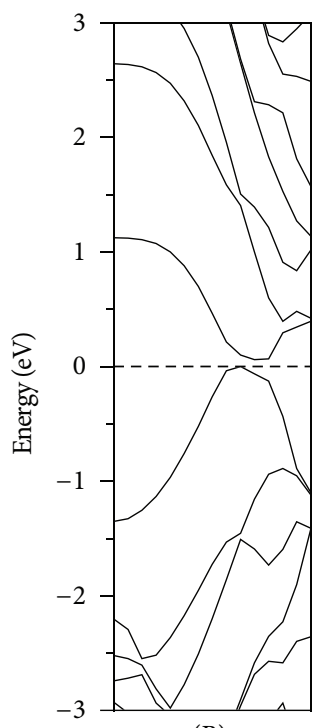

(B)

(a)

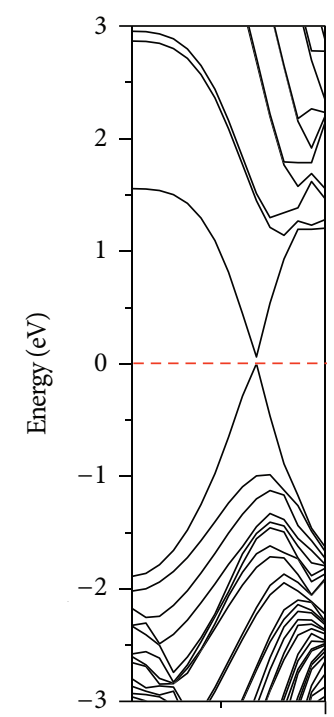

(B)
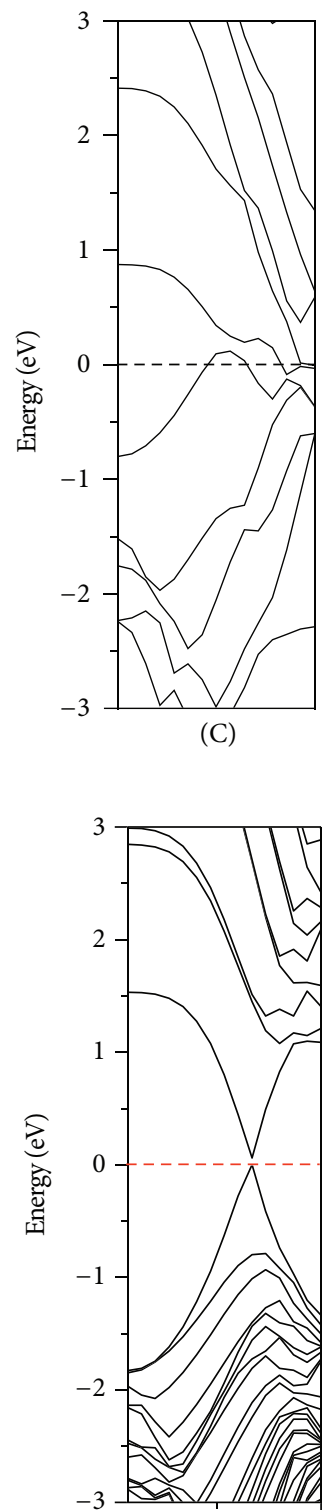

(C)

(b)

FIGURE 4: (a) Band structures of CNT(5,5) under an external electric field of (A) $F=0 \mathrm{~V} / \AA$, (B) $F=0.1 \mathrm{~V} / \AA \AA$, and $(\mathrm{C}) F=0.15 \mathrm{~V} / \AA$ along $y$-axis, respectively. (b) Band structures for $\operatorname{CNT}(5,5) @ B N N T(10,10)$ under $(\mathrm{A}) F=0 \mathrm{~V} / \AA$, (B) $F=0.1 \mathrm{~V} / \AA$, and $(\mathrm{C}) F=0.15 \mathrm{~V} / \AA$ along $y$-axis, respectively.

respectively, which are consistent with results of similar studies [28]. For the zigzag DWHNTs, $d_{i}$ affects the band gap of C/BN-DWHNTs. A different size $\operatorname{CNT}(7,0)$ was considered as the inner tube of the zigzag C/BN-DWHNTs. The calculated band gaps are listed in Table 1(b). All the zigzag CNT(7,0)@BNNT structures are found to be direct gap semiconductors with both the valence band top and conduction band bottom at the $\Gamma$ point. In Figure 3(a), analysis of the PDOS of $\mathrm{CNT}(7,0) @ \mathrm{BNNT}$ indicated that $p$ orbitals of the carbon atoms dominate the band near Femi level. However, for $\operatorname{CNT}(7,0) @ B N N T(14,0)$, the $p$ orbitals of the nitrogen atoms have a contribution to the bands near the Femi level, which also proved the stronger interaction when the $d_{i}$ strayed from $\sim 3.6 \AA$. Figure $3(\mathrm{~b})$ shows the DOS of $\mathrm{CNT}(7,0) @ \operatorname{BNNT}(14,0)$ and $\mathrm{CNT}(7,0) @ \operatorname{BNNT}(16,0)$ DWHNTs. The band gap of CNT $(7,0) @ B N N T(14,0)$ became small when comparing with the $\operatorname{CNT}(7,0) @ \operatorname{BNNT}(16,0)$ during the stronger intertube interactions.

After the CNTs were encapsulated into the BNNT, for the stable structure such as $\operatorname{CNT}(5,5) @ \operatorname{BNNT}(10,10)$, their geometries changed little. To study the effect of the transverse electric field on the electronic structure of the nanotubes, $F$ along $y$ direction (perpendicular to the tube axis) was imposed. We first examined the electronic properties of 
TABLE 1: (a) Calculated bond lengths $\left(a_{\mathrm{C}-\mathrm{C}}\right.$ and $\left.a_{\mathrm{B}-\mathrm{N}}\right)$ in the direction of the tube axis $(z)$ and in the perpendicular direction $(r)$, intertube spacing $\left(d_{i}\right)$, the unit being $\AA$, the binding energy $\left(E_{b}\right)$, and the forming energy $\left(E_{f}\right)$ in $\mathrm{kJ} / \mathrm{mol}$ of different geometrically optimized armchair double-wall hetero-nanotube optimizations. (b) Calculated bond lengths $\left(a_{\mathrm{C}-\mathrm{C}}\right.$ and $\left.a_{\mathrm{B}-\mathrm{N}}\right)$ in the perpendicular direction $(r)$, intertube spacing $\left(d_{i}\right)$, the unit being $\AA$, the binding energy $\left(E_{b} \mathrm{~kJ} / \mathrm{mol}\right)$ and the forming energy $\left(E_{f} \mathrm{~kJ} / \mathrm{mol}\right) \mathrm{kJmol}{ }^{-1}$, and GGA band gap $\left(E_{g}\right.$ eV) of different geometrically optimized zigzag double-wall hetero-nanotube optimizations. (c) The calculated bond lengths $\left(a_{\mathrm{C}-\mathrm{C}}\right.$ and $\left.a_{\mathrm{B}-\mathrm{N}}\right)$ of various doublewall hetero-nanotubes, in the direction of the tube axis $(z)$ and in the perpendicular direction $(r)$, and intertube spacing $\left(d_{i}\right)$ of different geometrically optimized double-wall hetero-nanotube optimizations; the unit is $\AA$.

(a)

\begin{tabular}{|c|c|c|c|c|c|c|c|}
\hline & \multicolumn{2}{|c|}{$a_{\mathrm{C}-\mathrm{C}}$} & \multicolumn{2}{|c|}{$a_{\mathrm{B}-\mathrm{N}}$} & \multirow{2}{*}{$d_{i}$} & \multirow{2}{*}{$E_{b}$} & \multirow{2}{*}{$E_{f}$} \\
\hline & $r$ & $z$ & $r$ & $z$ & & & \\
\hline CNT $(5,5) @ B N N T(8,8)$ & 1.385 & 1.418 & 1.540 & 1.471 & 2.60 & -761.89 & 12.537 \\
\hline CNT $(5,5) @ B N N T(9,9)$ & 1.420 & 1.434 & 1.473 & 1.453 & 2.97 & -773.74 & -0.493 \\
\hline CNT(5, 5)@BNNT $(10,10)$ & 1.435 & 1.441 & 1.439 & 1.441 & 3.45 & -774.41 & -2.450 \\
\hline CNT $(5,5) @ B N N T(11,11)$ & 1.441 & 1.443 & 1.438 & 1.441 & 4.08 & -772.29 & -1.455 \\
\hline CNT(5, 5)@BNNT(12, 12) & 1.436 & 1.440 & 1.444 & 1.442 & 4.83 & -769.60 & -0.023 \\
\hline CNT $(5,5) @ B N N T(13,13)$ & 1.434 & 1.440 & 1.448 & 1.444 & 5.54 & -768.83 & -0.013 \\
\hline $\operatorname{CNT}(6,6) @ \operatorname{BNNT}(10,10)$ & 1.416 & 1.431 & 1.473 & 1.453 & 3.03 & -777.50 & -0.641 \\
\hline CNT(6, 6)@BNNT(11, 11) & 1.433 & 1.440 & 1.446 & 1.445 & 3.49 & -778.17 & -2.689 \\
\hline CNT(6, 6)@BNNT(12, 12) & 1.440 & 1.444 & 1.439 & 1.441 & 4.15 & -775.66 & -1.376 \\
\hline $\mathrm{CNT}(7,7) @ B N N T(12,12)$ & 1.431 & 1.438 & 1.448 & 1.445 & 3.50 & -780.77 & -2.657 \\
\hline
\end{tabular}

(b)

\begin{tabular}{|c|c|c|c|c|c|c|}
\hline & $\begin{array}{c}a_{\mathrm{C}-\mathrm{C}} \\
r\end{array}$ & $\begin{array}{c}a_{\mathrm{B}-\mathrm{N}} \\
r\end{array}$ & $d_{i}$ & $E_{b}$ & $E_{f}$ & $E_{g}$ \\
\hline$\overline{\mathrm{CNT}}(6,0) @ \mathrm{BNNT}(13,0)$ & 1.439 & 1.466 & 2.947 & -763.81 & -0.173 & Metallic \\
\hline CNT $(6,0) @ B N N T(14,0)$ & 1.450 & 1.452 & 3.260 & -765.74 & -2.488 & Metallic \\
\hline CNT $(6,0) @ B N N T(15,0)$ & 1.450 & 1.447 & 3.601 & -765.55 & -2.678 & Metallic \\
\hline CNT $(6,0) @ B N N T(16,0)$ & 1.458 & 1.445 & 4.043 & -764.40 & -1.919 & Metallic \\
\hline CNT(6, 0)@BNNT $(17,0)$ & 1.458 & 1.443 & 4.412 & -762.85 & -0.705 & Metallic \\
\hline $\operatorname{CNT}(7,0) @ B N N T(14,0)$ & 1.431 & 1.466 & 2.987 & -768.25 & -0.271 & 0.146 \\
\hline $\mathrm{CNT}(7,0) @ \mathrm{BNNT}(15,0)$ & 1.440 & 1.453 & 3.240 & -769.60 & -2.457 & 0.247 \\
\hline CNT(7, 0)@BNNT $(16,0)$ & 1.446 & 1.446 & 3.670 & -769.50 & -2.685 & 0.302 \\
\hline $\operatorname{CNT}(7,0) @ B N N T(17,0)$ & 1.449 & 1.443 & 3.979 & -768.25 & -1.829 & 0.281 \\
\hline CNT $(7,0) @ B N N T(18,0)$ & 1.449 & 1.442 & 4.444 & -766.51 & -0.731 & 0.262 \\
\hline $\mathrm{CNT}(8,0) @ \mathrm{BNNT}(16,0)$ & 1.439 & 1.453 & 3.288 & -773.45 & -2.467 & 0.492 \\
\hline $\operatorname{CNT}(8,0) @ B N N T(17,0)$ & 1.444 & 1.446 & 3.636 & -772.49 & -2.581 & 0.558 \\
\hline CNT $(9,0) @ B N N T(17,0)$ & 1.436 & 1.453 & 3.305 & -775.66 & -2.395 & 0.034 \\
\hline CNT $(9,0) @ B N N T(18,0)$ & 1.443 & 1.445 & 3.627 & -775.09 & -2.608 & 0.027 \\
\hline CNT(10,0)@BNNT(18,0) & 1.434 & 1.452 & 3.368 & -777.78 & -2.471 & 0.805 \\
\hline CNT(10,0)@BNNT(19,0) & 1.441 & 1.444 & 3.603 & -777.11 & -2.571 & 0.811 \\
\hline CNT(11, 0)@BNNT $(20,0)$ & 1.442 & 1.444 & 3.610 & -776.15 & -2.558 & 0.814 \\
\hline CNT(11, 0)@BNNT $(21,0)$ & 1.441 & 1.442 & 3.976 & -777.30 & -1.799 & 0.808 \\
\hline
\end{tabular}

(c)

\begin{tabular}{lccc}
\hline \multicolumn{4}{c}{$\mathrm{CNT}(5,5) @ \mathrm{BNNT}(10,10)$} \\
\hline$E(\mathrm{~V} / \AA)$ & 0 & 0.1 & 0.25 \\
$a_{\mathrm{C}-\mathrm{C}}{ }_{r}$ & 1.435 & $1.433-1.437$ & $1.439-1.431$ \\
$a_{\mathrm{C}-\mathrm{C}}{ }^{z}$ & 1.435 & $1.434-1.436$ & $1.433-1.440$ \\
$a_{\mathrm{B}-\mathrm{N}}{ }_{r}$ & 1.439 & $1.434-1.440$ & $1.429-1.447$ \\
$a_{\mathrm{B}}{ }^{r}$ & 1.441 & $1.447-1.472$ & $1.438-1.508$ \\
$d_{i x}$ & 3.592 & 3.614 & 3.697 \\
$d_{i y}$ & 3.602 & 3.597 & 3.581 \\
\hline \multicolumn{4}{c}{$\mathrm{CNT}(5,5)$} \\
\hline$E(\mathrm{~V} / \AA)$ & 0 & 0.1 & 0.25 (distortion) \\
$a_{\mathrm{C}-\mathrm{C}}{ }_{r}$ & 1.425 & $1.424-1.428$ & $1.421-1.437$ \\
$a_{\mathrm{C}-\mathrm{C}}{ }^{r}$ & 1.426 & $1.426-1.431$ & $1.392-1.476$ \\
\hline$E(\mathrm{~V} / \AA)$ & 0 & $\mathrm{BNNT}(10,10)$ & 0.25 \\
\hline$a_{\mathrm{C}-\mathrm{C}}{ }^{r}$ & 1.436 & $1.435-1.437$ & $1.436-1.444$ \\
$a_{\mathrm{C}-\mathrm{C}}$ & 1.452 & $1.447-1.452$ & $1.432-1.457$ \\
\hline
\end{tabular}




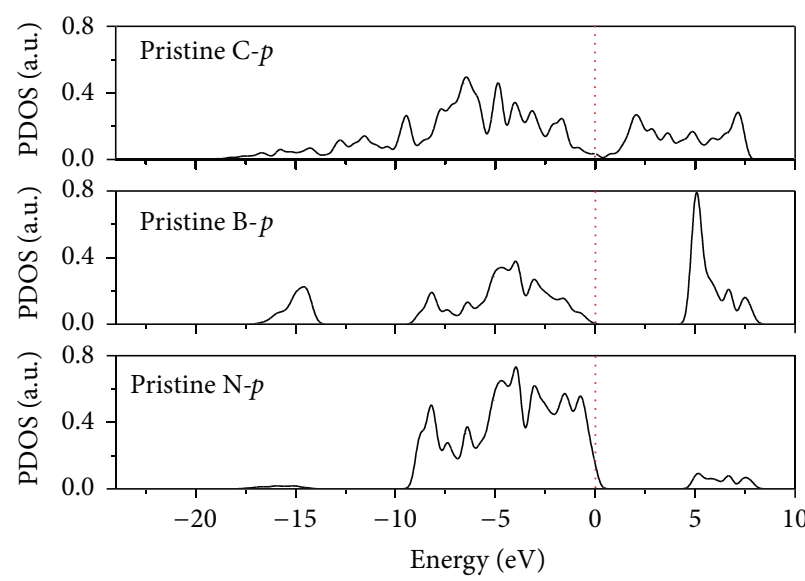

(a) $F=0 \mathrm{~V} / \AA$

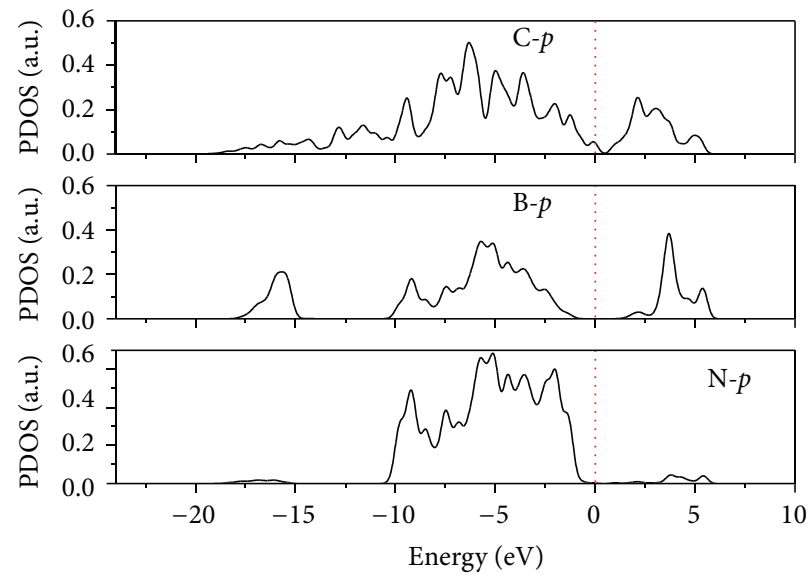

(c) $F=0 \mathrm{~V} / \AA$

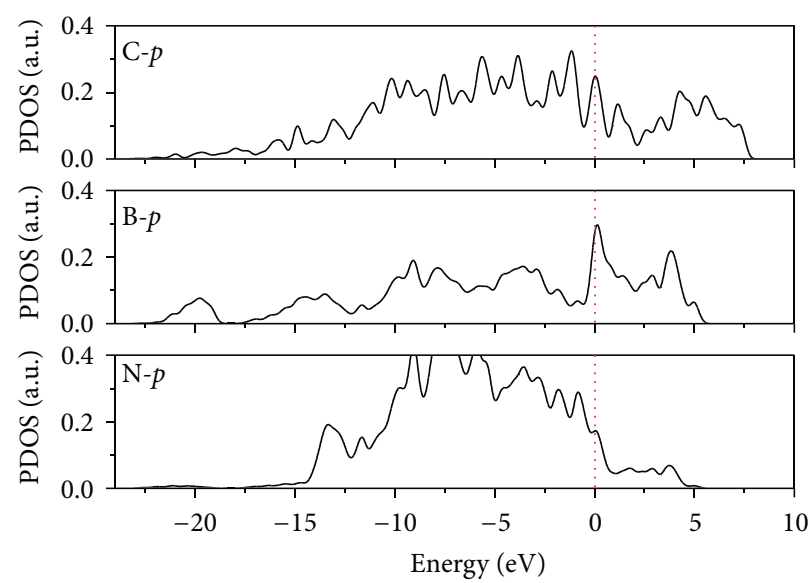

(b) $F=0.25 \mathrm{~V} / \AA$

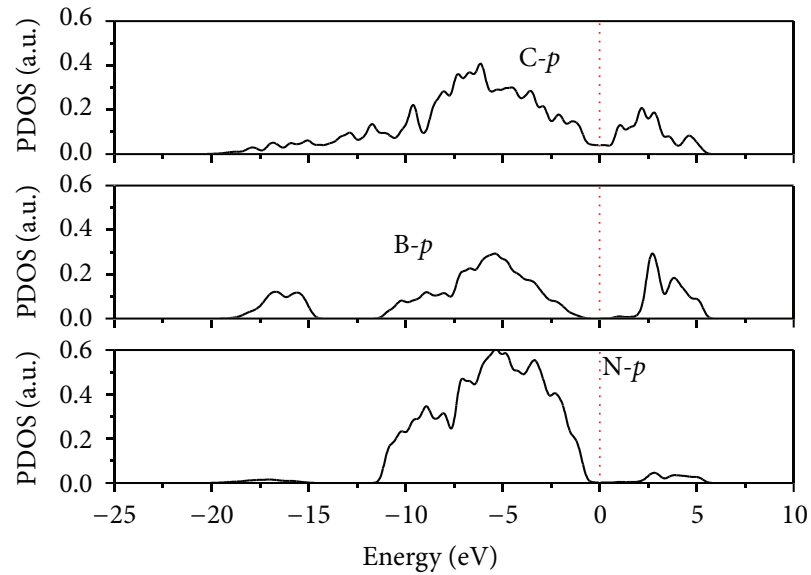

(d) $F=0.25 \mathrm{~V} / \AA$

Figure 5: PDOS of CNT $(5,5)$ and BNNT $(10,10)$ under an external electric field of (a) $F=0 \mathrm{~V} / \AA$ and $(\mathrm{b}) F=0.25 \mathrm{~V} / \AA \AA$, respectively. PDOS of $\mathrm{CNT}(5,5) @ B N N T(10,10)$ under an external electric field of (c) $F=0 \mathrm{~V} / \AA$ and $(\mathrm{d}) F=0.25 \mathrm{~V} / \AA$, respectively. The Fermi level lies at $0 \mathrm{eV}$ (dotted line). The solid line corresponds to $p$-state of $\mathrm{C}, \mathrm{B}$, and $\mathrm{N}$ at pristine $\mathrm{CNT}(5,5)$ and $\mathrm{BNNT}(10,10)$, respectively.

the $\operatorname{CNT}(5,5)$ under an electronic field (see Figure 4(a)). The $\operatorname{CNT}(5,5)$ remains semimetallic with an enhancement of density of states around the Fermi level with increasing the eternal electrical field. Two linear bands became flat (localized) around the Femi level in $\mathrm{CNT}(5,5)$ with increasing field strengths. It can therefore be inferred that the conductance will be greatly enhanced. And the results were well consistent with the literature [29]. The calculated values of the band structures for the armchair $\operatorname{CNT}(5,5) @ \operatorname{BNNT}(10,10)$ at $F=0,0.1$ and $0.15 \mathrm{~V} / \AA$ are presented in Figure $4(\mathrm{~b})$. There is no distinct difference between the electronic structures of the CNT $(5,5) @ B N N T(10,10)$ under different transverse electric fields. It shows that the transverse field does not affect the electric structures evidently. Whereas the band structures for $\operatorname{CNT}(5,5)$ under $F=0$ and $F=0.25 \mathrm{~V} / \AA$ show a striking contrast, the band structures for the $\mathrm{CNT}(5$, 5)@BNNT $(10,10)$ under $F=0$ and $F=0.25 \mathrm{~V} / \AA$ are quite similar. The same phenomenon has been found for CNT $(6,6) @ B N N T(10,10)$ and CNT $(7,7) @ B N N T(12,12)$ DWHNTs when $F$ is smaller than critical $F_{c}$ (here $F_{c}$ is a boundary when $F>F_{c}$, the band structure of armchair
CNT@BNNT would change abruptly, when $F<F_{c}$, and the band structure of armchair CNT@BNNT would keep original shape). See the results in Table 1(c); both CNT and BNNT single-walled nanotubes experience large structural changes after geometric optimization with increasing $F$. When $F=$ $0.25 \mathrm{~V} / \AA$, optimized $\mathrm{CNT}(5,5)$ was distortion.

However, with increasing $F$, the DOS of the CNT@BNNT is not a simple superposition of the DOS for the individual CNT and pristine BNNT under $F$. The peak heights for several particular states on the DOS are moderately strengthened or weakened due to the tube-tube interaction. To explore the origin of this phenomenon, the PDOS for $\mathrm{C}, \mathrm{B}$, and $\mathrm{N}$ in pristine $\operatorname{CNT}(5,5), \operatorname{BNNT}(10,10)$, and $\mathrm{CNT}(5,5) @ \operatorname{BNNT}(10$, 10) under electric fields $F=0$ and $0.25 \mathrm{~V} / \AA$ was plotted, respectively, as shown in Figure 5. The calculated PDOS indicates that the $p$-states of carbon atoms contribute mainly to the energy levels near the Femi level. The p-states of boron and nitrogen in pristine BNNT are very sensitive to the $F$. However, the change is not obvious in the heterostructure, which maybe caused by the effect of the tube-tube interaction. 
We also examined the electronic properties of the zigzag CNT (7, 0)@BNNT(16, 0) and CNT(10,0)@BNNT(18,0) under an electronic field. We found that with increasing $F$ the band gap of zigzag CNT $(7,0) @ B N N T(16,0)$ decreases gradually, and the phenomena are similar to the singlewall CNT $(7,0)$ under an electronic field. Though the band structure of zigzag CNT(10, 0)@BNNT $(18,0)$ also decreases gradually with increasing $F$, it is different from the single-wall $\mathrm{CNT}(10,0)$, because of the increasing intertube interactions.

\section{Conclusions}

The electronic structures of the double-wall heteronanotubes near the Fermi level are dominated by the p-electrons of carbon atoms; the band structure of the armchair DWHNTs is difficult to modulate with changing intertube distance. However, either changing intertube distance or imposing electric field is the efficient way to modulated the band structure of zigzag DWHNTs. Our results suggest an interesting avenue of exploring novel heterostructure of CNT@BNNT for potentially important applications in CNT@BNNT-based nanodevices.

\section{Conflict of Interests}

The authors declare that there is no conflict of interests regarding the publication of this paper.

\section{Acknowledgments}

This project was supported by the Natural Science Foundation of China (Grant no. 51402251), the Natural Science Foundation of Jiangsu Province of China (Grant no. BK20140471), and Talent Introduction Project of Yancheng Institute of Technology (nos. XKR2011009 and XKR2011001).

\section{References}

[1] C. R. Dean, A. F. Young, I. Meric et al., "Boron nitride substrates for high-quality graphene electronics," Nature Nanotechnology, vol. 5, no. 10, pp. 722-726, 2010.

[2] D. Usachov, V. K. Adamchuk, D. Haberer et al., "Quasifreestanding single-layer hexagonal boron nitride as a substrate for graphene synthesis," Physical Review B, vol. 82, no. 7, Article ID $075415,2010$.

[3] G. Giovannetti, P. A. Khomyakov, G. Brocks, P. J. Kelly, and J. Van Den Brink, "Substrate induced band gap in graphene on hexagonal boron nitride: $A b$ initio density functional calculations," Physical Review B, vol. 76, no. 7, Article ID 073103, 2006.

[4] J. Sławińska, I. Zasada, and Z. Klusek, "Energy gap tuning in graphene on hexagonal boron nitride bilayer system," Physical Review B, vol. 81, no. 15, Article ID 155433, 2010.

[5] A. Ramasubramaniam, D. Naveh, and E. Towe, "Tunable band gaps in bilayer graphene-BN heterostructures," Nano Letters, vol. 11, no. 3, pp. 1070-1075, 2011.

[6] R. Nakanishi, R. Kitaura, J. H. Warner et al., "Thin singlewall BN-nanotubes formed inside carbon nanotubes," Scientific Reports, vol. 3, article 1385, 2013.
[7] A. Gomathi, M. Ramya Harika, and C. N. R. Rao, "Urea route to coat inorganic nanowires, carbon fibers and nanotubes by boron nitride," Materials Science and Engineering A, vol. 476, no. 1-2, pp. 29-33, 2008.

[8] M. Das, A. K. Basu, S. Ghatak, and A. G. Joshi, "Carbothermal synthesis of boron nitride coating on PAN carbon fiber," Journal of the European Ceramic Society, vol. 29, no. 10, pp. 2129-2134, 2009.

[9] L. Chen, H. Ye, Y. Gogotsi, and M. J. McNallan, "Carbothermal synthesis of boron nitride coatings on silicon carbide," Journal of the American Ceramic Society, vol. 86, no. 11, pp. 1830-1837, 2003.

[10] S. Li, Y. F. Wu, W. Liu, and Y. H. Zhao, "Control of band structure of van der Waals heterostructures: silicene on ultrathin silicon nanosheets," Chemical Physics Letters, vol. 609, pp. 161-166, 2014.

[11] P. Avouris, R. Martel, V. Derycke, and J. Appenzeller, "Carbon nanotube transistors and logic circuits," Physica B: Condensed Matter, vol. 323, no. 1-4, pp. 6-14, 2002.

[12] C. L. Phillips, C. S. Yah, S. E. Iyuke, K. Rumbold, and V. Pillay, "The cellular response of Saccharomyces cerevisiae to multi-walled carbon nanotubes (MWCNTs)," Journal of Saudi Chemical Society, vol. 19, no. 2, pp. 147-154, 2015.

[13] K. Chikkadi, M. Muoth, W. Liu, V. Maiwald, and C. Hierold, "Enhanced signal-to-noise ratio in pristine, suspended carbon nanotube gas sensors," Sensors and Actuators, B: Chemical, vol. 196, pp. 682-690, 2014.

[14] A. Freitas, S. Azevedo, and J. R. Kaschny, "Effects of a transverse electric field on the electronic properties of single- and multiwall BN nanotubes," Solid State Communications, vol. 153, no. 1, pp. 40-45, 2013.

[15] C.-W. Chen, M.-H. Lee, and S. J. Clark, "Band gap modification of single-walled carbon nanotube and boron nitride nanotube under a transverse electric field," Nanotechnology, vol. 15, no. 12, pp. 1837-1843, 2004.

[16] P. Ordejón, E. Artacho, and J. M. Soler, "Self-consistent order-N density-functional calculations for very large systems," Physical Review B, vol. 53, no. 16, Article ID R10441, 1996.

[17] D. Sánchez-Portal, P. Ordejón, E. Artacho, and J. M. Soler, "Density-functional method for very large systems with LCAO basis sets," International Journal of Quantum Chemistry, vol. 65, no. 5, pp. 453-461, 1997.

[18] J. P. Perdew, K. Burke, and M. Ernzerhof, "Generalized gradient approximation made simple," Physical Review Letters, vol. 77, no. 18 , pp. 3865-3868, 1996.

[19] H. J. Monkhorst and J. D. Pack, "Special points for Brillouinzone integrations," Physical Review B, vol. 13, no. 12, pp. 5188$5192,1976$.

[20] B. Delley, "An all-electron numerical method for solving the local density functional for polyatomic molecules," The Journal of Chemical Physics, vol. 92, no. 1, pp. 508-517, 1990.

[21] B. Delley, "From molecules to solids with the $\mathrm{DMol}^{3}$ approach," Journal of Chemical Physics, vol. 113, no. 18, pp. 7756-7764, 2000.

[22] J. P. Perdew and Y. Wang, "Accurate and simple analytic representation of the electron-gas correlation energy," Physical Review B, vol. 45, no. 23, pp. 13244-13249, 1992.

[23] S. Li, Y. Wu, Y. Tu et al., "Defects in silicene: vacancy clusters, extended line defects, and di-adatoms," Scientific Reports, vol. 5, article 7881, 2015.

[24] W. Liu, A. Tkatchenko, and M. Scheffler, "Modeling adsorption and reactions of organic molecules at metal surfaces," Accounts of Chemical Research, vol. 47, no. 11, pp. 3369-3377, 2014. 
[25] W. Liu, S. N. Filimonov, J. Carrasco, and A. Tkatchenko, "Molecular switches from benzene derivatives adsorbed on metal surfaces," Nature Communications, vol. 4, article 2569, 2013.

[26] W. Liu, J. Carrasco, B. Santra, A. Michaelides, M. Scheffler, and A. Tkatchenko, "Benzene adsorbed on metals: concerted effect of covalency and van der Waals bonding," Physical Review B, vol. 86, no. 24, Article ID 245405, 2012.

[27] J. Yuan and K. M. Liew, "Structural stability of a coaxial carbon nanotube inside a boron-nitride nanotube," Carbon, vol. 49, no. 2, pp. 677-683, 2011.

[28] V. Zólyomi and J. Kürti, "First-principles calculations for the electronic band structures of small diameter single-wall carbon nanotubes," Physical Review B, vol. 70, no. 8, Article ID 85403, 2004.

[29] S. V. Tishchenko, "Electronic structure of zigzag carbon nanotubes," Low Temperature Physics, vol. 32, no. 10, pp. 953-956, 2006. 

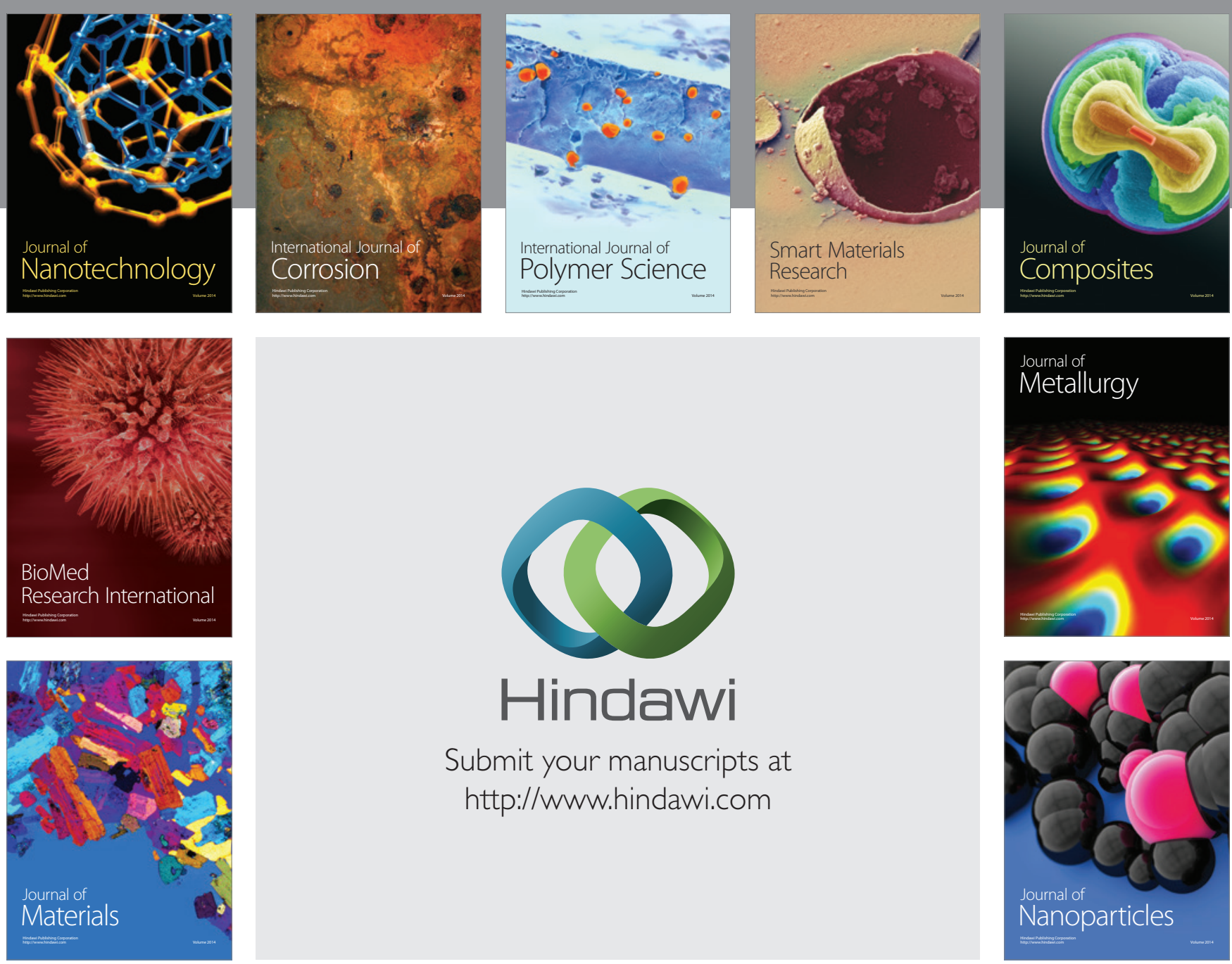

Submit your manuscripts at http://www.hindawi.com
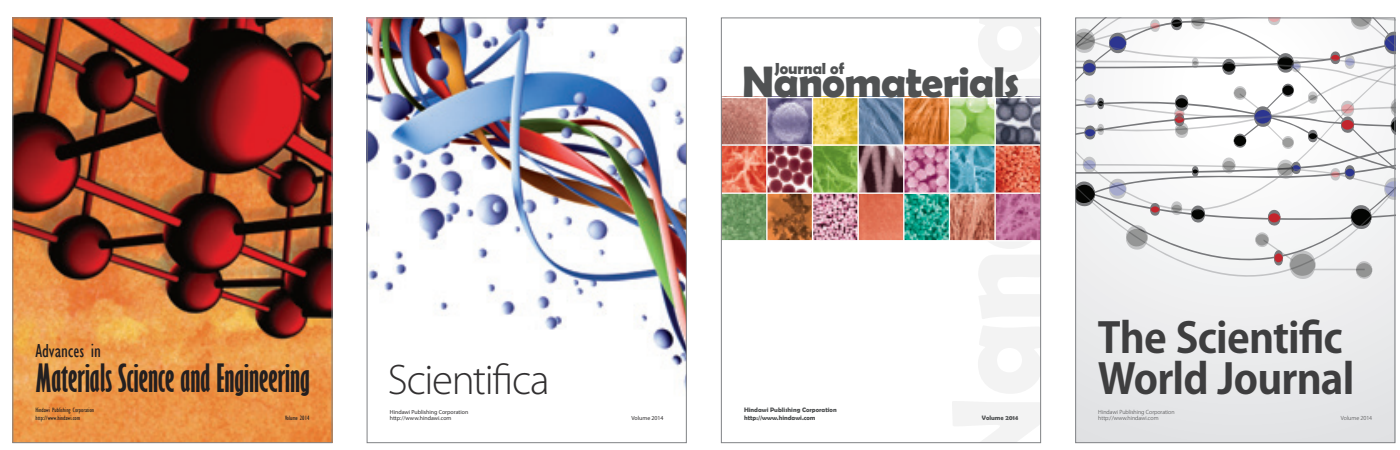

\section{The Scientific World Journal}
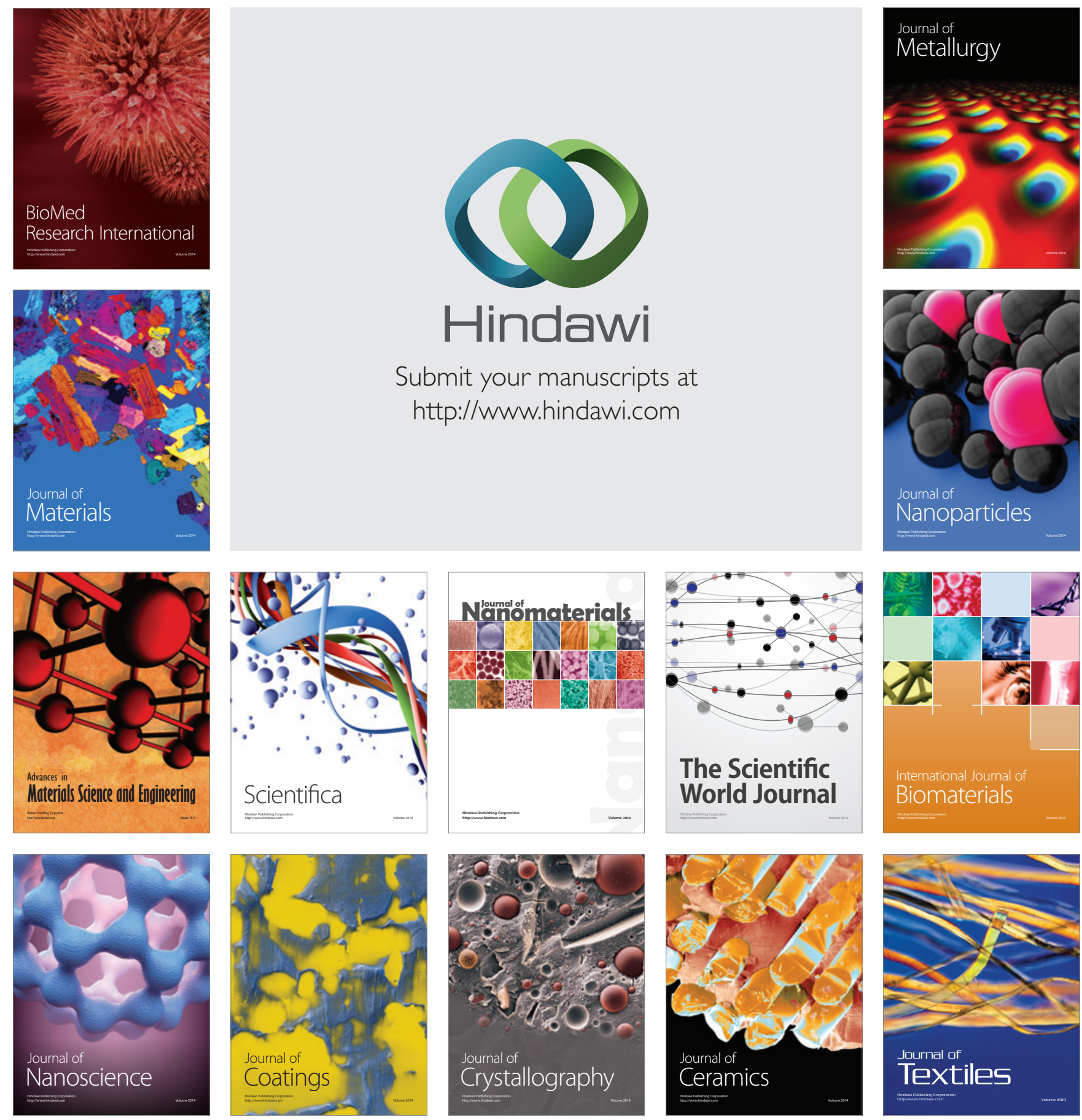